\title{
Large fibroid complicating pregnancy: a case report
}

\section{Lingampalli Naga Saketha*, Lopamudra B. John}

Department of Obstetrics and Gynaecology, MGMCRI, Pondicherry, India

Received: 03 March 2021

Revised: 09 April 2021

Accepted: 12 April 2021

\section{*Correspondence:}

Dr. Lingampalli Naga Saketha,

E-mail: drsaketha89@gmail.com

Copyright: () the author(s), publisher and licensee Medip Academy. This is an open-access article distributed under the terms of the Creative Commons Attribution Non-Commercial License, which permits unrestricted non-commercial use, distribution, and reproduction in any medium, provided the original work is properly cited.

\section{ABSTRACT}

Uterine fibroids are the most common benign tumors of the female reproductive system and are known to occur in $0.1-10.7 \%$ of pregnant women and incidence increases with increasing maternal age. Fibroids when present are associated with complications in $10-40 \%$ of pregnant patients like abdominal pain, spontaneous abortion, fetal malposition, placental abruption, premature rupture of membranes, cesarean deliveries, postpartum hemorrhage, preterm delivery and low birth weight infants.

Keywords: Uterine fibroids, Benign tumor, Caesarean deliveries

\section{INTRODUCTION}

Uterine fibroids are the most common benign tumors of the female reproductive system. The incidence of fibroids in pregnancy ranges from $0.1-10.7 \%$ of all pregnant women and incidence increases with increasing maternal age. ${ }^{1}$ The incidence of fibroids during pregnancy is likely to increase in the coming years, in association with delay in childbearing.

The most important factors in determining morbidity in pregnancy include fibroid number, size, location and relationship to placenta implantation.

Fibroids may negatively affect fertility and outcome of pregnancy. As most of fibroids are asymptomatic, the prevalence of fibroids may be greatly higher. Fibroids have been complicated by changes like degeneration leading to abdominal pain whose severity is varied from mild to acute abdomen. Fibroids are associated with antepartum, intrapartum and postpartum complications. Fibroids when present are associated with complications in $10-40 \%$ of pregnant patients like abdominal pain, spontaneous abortion, fetal malposition, placental abruption, premature rupture of membranes, increased rate of caesarean deliveries, postpartum hemorrhage, preterm delivery and low birth weight infants.

\section{CASE REPORT}

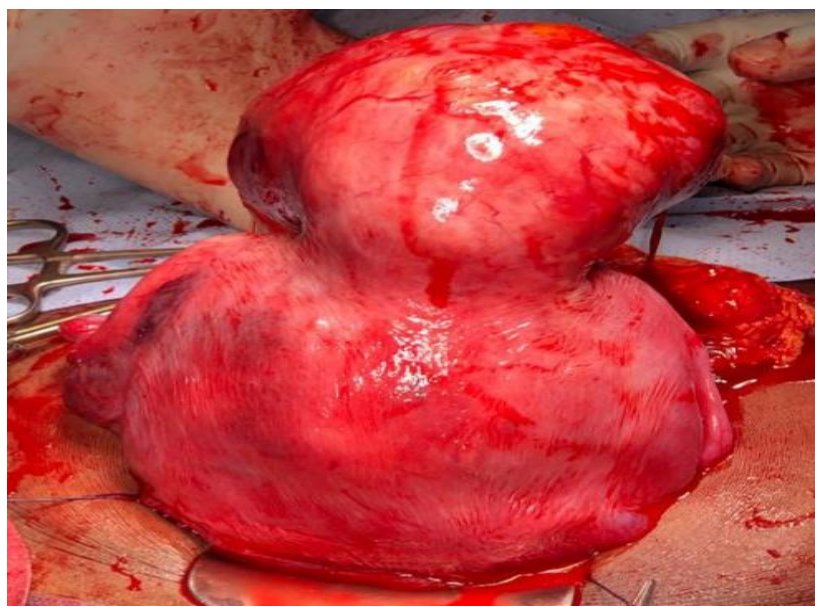

Figure 1: Fundal fibroid.

A 36 years elderly primi with bronchial asthma was referred as fibroid complicating pregnancy (fundal fibroid 
$9.6 \times 7.5 \times 11.6 \mathrm{~cm})$ at 13 weeks with complaints of abdominal pain on and off. She was on regular follow up. There was increase in size of fibroid since the time of diagnosis to delivery. She was given prophylactic steroids and started on nifedipine tocolysis from 32 weeks onwards. Tocolytic drugs were gradually tapered after 35 weeks when pain subsided. At 36 weeks she went into labor and emergency caesarean section with myomectomy done. She delivered a healthy baby of weight $2.1 \mathrm{~kg}$ with good Apgar. Intraoperatively-fundal subserosal fibroid of $10 \times 8 \mathrm{~cm}$ was present. Intraoperatively and post-operatively there were no complications and patient was discharged after one week. Histopathology was leiomyoma with focal hyaline change.

\section{DISCUSSION}

Most fibroids do not grow in pregnancy, but about $30 \%$ may enlarge during the first three months. Fibroids are usually asymptomatic. Pain is the most common complication in pregnancy and is seen most often in large fibroids $(>5 \mathrm{~cm})$ during the second and third trimesters. ${ }^{2}$ Localized abdominal pain can occur if a fibroid undergoes red degeneration, torsion (seen most commonly with a pedunculated subserosal fibroid) or impaction. Uterine fibroids are linked to an increased rate of spontaneous miscarriage, preterm labor, foetal malpresentation, labor dystocia, caesarean section postpartum haemorrhage and hysterectomy. ${ }^{3}$

The symptoms can be managed mostly with conservative treatment. A caesarean section is advisable for those who have previously had a myomectomy if the uterine cavity was opened.
Hysterectomy is indicated when there is substantial bleeding, pelvic pain or pressure or anemia refractory to iron replacement in women not wishing to preserve fertility.

\section{CONCLUSION}

Most of the fibroids in pregnancy are asymptomatic but may be associated with some complications affecting the course of pregnancy and labor depending on their size and location. So, pregnancy has to be cautiously screened in the antenatal period, through regular follow-up, to detect any adverse obstetric complications and so improve the outcome. . The use of ultrasonography has simplifed diagnosis and management of fbroids in pregnancy.

\section{Funding: No funding sources \\ Conflict of interest: None declared \\ Ethical approval: Not required}

\section{REFERENCES}

1. Lee HJ, Norwitz ER, Shaw J. Contemporary management of fibroids in pregnancy. Rev Obstet Gynecol. 2010;3(1):20-7.

2. Pandya M, Senta J, Shah S, Patel D, Khanderiya K. A rare case of multiple fibroids with pregnancy. Indian J Obstet Gynecol Res. 2018;5(2):308-10.

3. Othman M, Alzahrani A. Huge fibroid at term pregnancy: case report and review of literature. Gynecol Obstet. 2015;6(5).

Cite this article as: Saketha LN, John LB. Large fibroid complicating pregnancy: a case report. Int $\mathrm{J}$ Reprod Contracept Obstet Gynecol 2021;10:2099100 . 